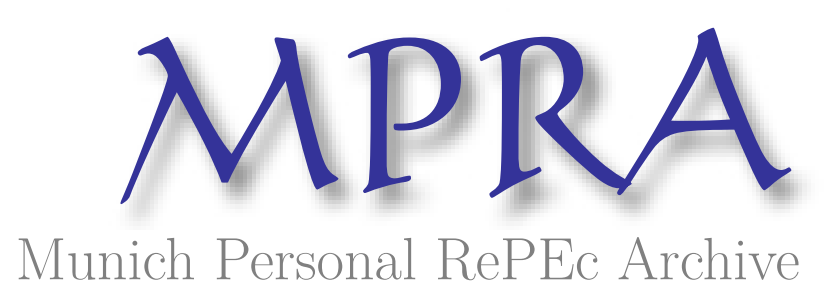

\title{
Some Propositions on Intergenerational Risk Sharing, Social Security and Self-Insurance
}

Aoki, Takaaki

State University of New York at Buffalo, Department of Economics

August 2006

Online at https://mpra.ub.uni-muenchen.de/11684/

MPRA Paper No. 11684, posted 24 Dec 2008 08:40 UTC 


\title{
Some Propositions on Intergenerational Risk Sharing, Social Security and Self-Insurance*
}

\author{
Takaaki Aoki**
}

* JEL classification: A1, D0, D8, H0, J0. This paper is written by summarizing a part of results obtained in a series of my previous working papers: Aoki, Takaaki (2003)

** State University of New York at Buffalo, Department of Economics, 415 Fronczak Hall, North Campus, Buffalo, NY 14260 USA

Tel: (716)645-2121Ｅ-mail: taoki@acsu.buffalo.edu 


\begin{abstract}
This article describes, within a myopic intergenerational bargaining framework incorporating two discrete periods and binary states of risks, some new aspects regarding the mixture of intergenerational risk sharing and social security. Here, state-dependent utility under mortality risk proves to generate parents' peculiar indifference curve regarding insurance contract, and self-insurance is shown to play a crucial role on the decision regarding social security holding and intergenerational transfer contract. This peculiar aspect, given for the first time in this article, also derives some novel features of insurance theory under lifetime uncertainty, where the current position in social security contract could adversely affect parents' decision regarding intergenerational risk sharing with children. In addition, other basic results regarding the sensitivity to default risk and taxation in social security are summarized.
\end{abstract}

\title{
1. Introduction
}

The objective of this article is simply and clearly to describe some new economic aspects of intergenerational risk sharing under lifetime uncertainty within a myopic bargaining framework. Atkinson and Stiglitz (1980) and Obstfeld and Rogoff (1996) are textbooks of public economics and international macroeconomics, especially the latter of which contains the description of a risk sharing with default risk and saving. Ehrlich and Becker (1972) develop a basic theory of demand for insurance that emphasizes the interaction between market insurance, "self-insurance" and "self-protection". For some other examples among related literatures, Shiller (1999), Ball and Mankiw (2001), Enders and Lapan (1982) and Gordon and Varian (1988) examine the economic role of intergenerational risk sharing. Yaari (1965) is a classical article, which pursuits the implications of life insurance under the 
mortality risk. Hayashi, Altonji and Kotlikoff (1996) is an empirical work on intra-gamily \& inter-generational risk sharing accompanying the possibility of self-insurance. Analyses on bequest motives appear, among many, in Abel (1985, 1987), Hurd (1989), Bernheim, Shleifer and Summers (1985) and Bernheim (1991).

In this article we newly focus on a peculiar shape of parents' indifference curve, which arises from a state dependent aspect of utility under a life uncertainty environment, using a model of two discrete periods and binary states of mortality/income risks. In section 2, a basic framework is set, in which two adjacent generations, parents in old adulthood and children in young adulthood are facing the decision regarding intergenerational risk sharing with/without an available old-age social security for parents. In section 3, some characteristics in parents' difference curve are explained, where self-insurance plays an important role on the insurance contract decision. On the basis of these analyses, we claim some fundamental propositions regarding the optimal allocation of social security and intergenerational risk sharing in section 4 , and some regarding the sensitivity to default risk and taxation in social security in section 5.

\section{Basic setting}

At first we divide each generation's lifetime roughly into three stages, " $Y$ " (for the child), " $M$ " (for the young adulthood) and " $O$ " (for the old adulthood), each of which corresponds with each discrete period $(\doteqdot 30$ years). Assume that, at the beginning of period $t$, two adjacent generations, " $p$ " (parents) and " $c$ " (children), are now going to begin stage $O$ and $M$, respectively. ${ }^{1}{ }^{2}$ Parents hold an available asset $A^{p}$, and children's disposable income is $W^{c}$. During this period, there exist two types of binary risk, the risk of death

1 This is a typical overlapping generation model.

2 These notations are also used in superscripts/subscripts. 
(mortality risk $\left.s_{1}(=1,0)\right)$ for parents, and that of disposable income $s_{2}(=1,0)$ for children. The risk of death exists is revealed exactly at the middle point of stage $O$, when they are alive $\left(s_{1}=1\right)$ with probability $\varphi$, or die $\left(s_{1}=0\right)$ with probability $1-\varphi$. The income risk is revealed exactly at the middle point of stage $M$, when they earn the higher income $W_{1}^{c}$ $\left(s_{2}=1\right)$ with probability $\varphi^{\prime}$, or the lower income $W_{0}^{c}\left(s_{2}=0\right)$ with $1-\varphi^{\prime}$. Therefore, the revelation of mortality risk for parents, and that of income risk for children exactly coincide with each other in time. Each generation $i(=p, c)$ holds an egoistic utility, which depends explicitly only on its own consumption only during stage $M$ and $O$, not during $Y$, and takes a form of state-dependent utility:

$$
\begin{array}{ll}
\tilde{u}^{i}=u^{i}\left(c_{M 1}^{i}\right)+\beta u^{i}\left(c_{M 2}^{i}\right)+\beta^{2}\left\{u^{i}\left(c_{O 1}^{i}\right)+\beta u^{i}\left(c_{O 2}^{i}\right)\right\} & \text { if } s_{1}=1 \\
\tilde{u}^{i}=u^{i}\left(c_{M 1}^{i}\right)+\beta u^{i}\left(c_{M 2}^{i}\right)+\beta^{2} u^{i}\left(c_{O 1}^{i}\right) & \text { if } s_{1}=0 \\
u^{i}(c)=c^{1-\sigma} /(1-\sigma) \text { if } \sigma \neq 1, \text { or } u^{i}(c)=\ln c \text { if } \sigma=1
\end{array}
$$

Here $\beta$ is a constant time preference for each half period, $\sigma$ is a constant relative risk aversion coefficient, $c_{M 1}^{i}$ is a real consumption of generation $i$ during the first half period of stage $M, c_{O 2}^{i}$ is a real consumption of generation $i$ during the second half period of stage $O$, or etc. Utility function $u(\cdot)$ is increasing and concave, and assumes ordinary Inada conditions. The real interest rate for each half period is denoted by $r .{ }^{3}$ Children's life strategies during stage $M$ (number of children to bear $N$, human capital investment for each child $H$, asset plan $A$ ) are exogenously given, except for the intergenerational transfer contracts with parents $(S, B)$. For parents, there are two options of old adulthood insurance for mortality risk, intergenerational transfer with children $(S, B)$ and social

${ }_{3} \beta=1 /(1+r)$ is assumed just for convenience but without loss of generality. 
security $(R, P) .{ }^{4}{ }^{5}$ If these transfer contracts are actuarially fair, they necessarily satisfy:

$$
l_{1}: \varphi S=(1-\varphi) B \text { or } l_{1}: \varphi R=(1-\varphi) P
$$

At first assume that $s_{1}$ and $s_{0}$ are uncorrelated. Then the associated indirect utilities for parents and children regarding a transfer contract schedule $(S, B)$ are represented as:

$$
\begin{aligned}
& \widetilde{v}^{p}\left(S, B, \varphi, \beta, r, A^{p}\right) \equiv u\left(A^{p}-B\right)+\varphi \beta u((1+r)(S+B)) \\
& \widetilde{v}^{c}\left(S, B, \varphi, \beta, r, W^{c}\right) \equiv \max _{c_{1}}\left(\begin{array}{l}
\varphi\left[u\left(c_{1}\right)+\beta u\left((1+r)\left(W^{c}-S-c_{1}\right)\right)\right] \\
+(1-\varphi)\left[u\left(c_{1}\right)+\beta u\left((1+r)\left(W^{c}+B-c_{1}\right)\right)\right]
\end{array}\right)
\end{aligned}
$$

\section{Some peculiar aspects of intergenerational risk sharing}

See Figure 1. The dotted line $a, c$, and $d$ are the indifference curves of parents, which draws the contour lines $\widetilde{v}^{p}\left(S, B, \varphi, \beta, r, A^{p}\right)=\bar{v}^{p}$ for distinct constants, $\bar{v}^{p}$ 's. On the other hand, $b, e$, and $f$ are those of children, which draws the contour lines $\widetilde{v}^{c}\left(S, B, \varphi, \beta, r, \overline{\bar{W}}^{c}\right)=\bar{v}^{c}$ for distinct $\bar{v}^{c}$ 's. Under the settings of section 2 , there exists a set of intergenerational transfer contract $(S, B)$, such that: (1) both parents and children are willing to conclude the contract. (Participation constraints), and (2) each contract is Pareto-optimum. (Pareto optimality conditions) Furthermore, the compact set denoted by $\underline{X}_{p}$, satisfying the above conditions (1) and (2), is located inside the area $S>0, B>0$

${ }_{4}^{4}(S, B)$ and $(R, P)$ denote (Support,Bequest) and (Re ceipt, Payment), respectively. Here the left-hand side of $(S, B)$ and $(R, P)$ denotes receipt with occurrence of $s_{1}=1$, and the right-hand side does payment with $s_{1}=0$, both sides measured by present value at the beginning of the period.

${ }^{5}$ In this article, we do not set any substantial distinction between social security and market insurance.

${ }^{6}$ If social security program $(R, P)$ is also available, then:

$\widetilde{v}^{p}\left(S+R, B+P, \varphi, \beta, r, A^{p}\right) \equiv u\left(A^{p}-B-P\right)+\varphi \beta u((1+r)(S+R+B+P)) \quad\left(2.4 \mathrm{a}^{\prime}\right)$

${ }^{7}$ Under no correlation between $S_{1}$ and $S_{0}, W^{c}$ is defined as the expected disposable income, that is $W^{c} \equiv \varphi^{\prime} W_{1}^{c}+\left(1-\varphi^{\prime}\right) W_{0}^{c}$. 
and $(1-\varphi) B>\varphi S . \underline{X}_{p}$ is a bold segment line $G F$, where point $F$ is a tangent point of parents' indifference curve $a$, and that of children $e$, and point $G$ is a tangent point of children's indifference curve $b$ and that of parents $d$. Any indifference curves of parents ( $a, c$ or $d$ ) are shown to be tangent with two lines, $B=A^{p}$ and certainty line $l_{2}: B=-S$. Children, if they do not conclude any transfer contract, will be undoubtedly at point $O$ (that is, $(S, B)=(0,0))$, being tangent with the actuarially fair line $l_{1}: \varphi S=(1-\varphi) B$. In general, as illustrated in the indifference curve $f$, any arbitrary indifference curve of children is tangent with the constant premium line $l_{1}^{\prime}:(1-\varphi) B-\varphi S=k$, at the intersection of $l_{1}^{\prime}$ and $l_{2}: B=-S$.

See Figure 2. Now we examine parents' position within a given transfer contract scheme $(S, B)$. If parents are not given any transfer contract, their position is illustrated as point $D:(S, B)=\left(0,\left(\varphi^{1 / \sigma} \beta A^{p}\right) /\left(1+\varphi^{1 / \sigma} \beta\right)\right)$, where $a$ is tangent with the horizontal axis $S=0$, exactly at point $D .{ }^{8}$ Here $B$ is not an amount of bequest, but is some conditional cost on death $\left(s_{1}=0\right)$ to be additionally discarded as a result of partially self-insuring mortality risk $s_{1} \cdot{ }^{9}$ Thus point $D$ is an optimal "self-insurance (self-contract)", which parents would choose when the social security is not available. Instead, if parents make the actuarially fair and flexible social security contract, their position is point $E:(R, P)=\left((1-\varphi) \beta A^{p} /(1+\varphi \beta), \varphi \beta A^{p} /(1+\varphi \beta)\right)$, where their indifference curve $c$ is tangent with $l_{1}: \varphi R=(1-\varphi) P$, at point $E . .^{10}$ See point $I$ and $J$, both located on $l_{1}$. $I$ is the point where $a$, which passes point $D$, intersects with $l_{1}$. Therefore, $I$ is a

\footnotetext{
${ }^{8}$ In this case, parents' maximization problem is equivalent with maximizing their indirect utility associated with the transfer contract $(S, B),(2.4 \mathrm{a})$, with regard to $B$, keeping $S$ fixed at 0 .

9 This cost is paid along axis $B$.

10 Note that parents' maximization problem is equivalent with maximizing their associated indirect utility (2.4a), with regard to $(S, B)$ satisfying $l_{1}$. Here intergenerational transfer $(S, B)$ is replaced with the notation for social security contract $(R, P)$.
} 
reservation actuarially fair contract, which assures a minimum utility, same as an "optimal self-insuring contract" $D$. On the other hand, $J$ is a point on $l_{1}$, at which an indifference curve of parents takes a minimum in $S$ exactly at $J$. Now we consider some fixed actuarially fair contract on $l_{1}$, represented as point $K:\left(R_{K}, P_{K}\right) .{ }^{11}$ Assume that $K$ is located on between point $O$ and $J$. In this case, parents can be even better off than at $K$, by discarding some additional cost $P^{\prime}$ say, conditionally on death (along the axis in $B$ ), as a kind of self-insuring contract. Let point $K^{\prime}$ be the tangent point of parents' indifference curve and $S=R_{K}$. Then the optimal additional cost $P^{\prime}$, which parents should discard conditionally on death, is calculated as distance $K K^{\prime}$. If $K$ is located in the upper-right of $J$ along $l_{1}$, then parents do not have to pay any additional cost along in $B$. The overall locus of a mixed contract schedule $\widetilde{K}$ say, which should include that additional and conditional cost in correspondence with each given contract $K$, would be a semi-segment of line, $D J E Q$, as drawn in a bold line in Figure 1.12 we denote this set, which can be optimally attained as a result of making use only of an actuarially fair contract set, by $\underline{X}_{a c} \cdot{ }^{13}$

Without any contracts concluded, parents would stand at point $D$, while children would at a different point $O$. This aspect makes it for both parents and children impossible to set initially some value for the state contingent claim between two states of $s_{1}$, or equivalently to set the initial relative price between $S$ and $B$. This is a totally different point from Arrow-Debreu state-contingent exchange economy, in which state contingent claim (or state price) enables them to arrive at a market-clearing and Pareto optimal

\footnotetext{
${ }^{11} R_{K} \geq 0, P_{K} \geq 0$.

12 Point $Q$ on $l_{1}$ is infinitely far in the upper-right side.

${ }^{13}$ Clearly $\widetilde{K}$ coincides with $K^{\prime}$ if $K$ is located on a segment line $O J$, and coincides with $K$ itself if $K$ is on a semi-segment line $J Q$.
} 
equilibrium. ${ }^{14}$ Therefore, in this myopic bargaining frame work, an automatic price adjustment process to a unique equilibrium point on $\underline{X}_{p}$ cannot be expected, as far as any additional restriction (e.g., regarding the altruistic weight in utility between parents and children) or any other peculiar agreements or algorithms are not introduced. ${ }^{15}$ This is one important economic feature of intergenerational contract curve $\underline{X}_{p}$. On the other hand, with a fixed level of available social security, for example $K=J$, in which self-insurance is not necessary, Arrow-Debreu state-contingent exchange economy can be well defined. In this case, an equilibrium (Pareto optimal and market clearing) contract does not depend on the existence of altruism between parents and children, since, in general, the weight of altruism does not transform the shape of extended contract curve, which is drawn just by relaxing participation constraints. ${ }^{16}$

\section{Some propositions regarding the mixture of intergenerational risk sharing and social} security

Now we compare, within the current framework, actuarially fair social security and intergenerational transfer contract, from parents' viewpoint. Especially one important question is: Do parents choose only an actuarially fair social security or only an intergenerational transfer contract with children, or both of them? Although it depends on where an available social security $K$ and an available intergenerational transfer $Y$ are

14 If both parents and children agree with standing initially at point $O$, there does exist a competitive equilibrium on $\underline{X}_{p}(G F)$.

15 One example is one-sided (or two-sided) altruistic utility of the form $U^{p}=\widetilde{u}^{p}+\psi\left(\widetilde{u}^{c}\right)$.

16 With a fixed social security $\left(R_{K}, P_{K}\right)$, this extended contract curve (not $\left.\underline{X}_{p}\right)$ is drawn by a set of points, where parents' indifference curve is tangent with the curve generated by shifting children's indifference curve in parallel along with $l_{1}$ by vector $\left(R_{K}, P_{K}\right)$. 
located on $l_{1}$ and on $\underline{X}_{p}$ respectively, some aspects regarding this question can be extracted, by setting one simple assumption regarding children's behavior, that they would accept any intergenerational transfer which is offered from parents, if it assures at least the same utility as at point $Y$ in terms of children's associated indirect utility (2.4b). ${ }^{17}$ Denote parents' maximized utilities, which can be attained by concluding only social security $K$, only intergenerational transfer $Y$, both of them, by $V^{p}(K), V^{p}(Y)$ and $V^{p}(K+Y)$, respectively. ${ }^{18}$ At first, we claim a following proposition and corollary.

Proposition 1: See Figure 2. Then:

(i) Assume that $Y$ coincides with $G$, the point which attains parents' maximum utility on $\underline{X}_{p}$. Then, for any arbitrary $K$, which is located on the segment line of $l_{1}, O Z$, it holds that $V^{p}(Y) \leq V^{p}(K+Y)$ and $V^{p}(K) \leq V^{p}(K+Y)$.

(ii) Assume that $Y$ coincides with $F$, the point which attains parents' minimum utility on $\underline{X}_{p}$. Then, for any arbitrary $K$ on $l_{1}$ such that $O K \leq O^{\prime} Z^{\prime \prime}$, it holds that $V^{p}(Y) \leq V^{p}(K+Y)$ and $V^{p}(K) \geq V^{p}(K+Y)$.

Corollary 1: Consider an already concluded (mandatory) intergenerational transfer $Y$ on $\underline{X}_{p}$. Then, any arbitrary social security $K$ on $l_{1}(S \geq 0, B \geq 0)$ surely enhances parents' indirect utility without any necessity to discard any additional and conditional cost, if $K$ is not extremely large in amount. This always holds whether there exists some correlation between parents' state $s_{1}$ and children's state $s_{2}$ or not.

17 Therefore we assume implicitly that children do not enter any other transfer contract including social security.

18 With each of these three options, parents may pay, if necessary, an additional and conditional cost along the axis in $B$ (self-insurance), as explained in Section 3. For rigorous formulation of parents' problems to be solved, see Appendix. 
Corollary 1 is clear from children's indifference curve under positive/negative correlation between $s_{1}$ and $s_{2}$, as shown in Figure 3 and 4 . Proposition 1 has quite interesting economic implications. First, parents, together with a mandatory intergenerational transfer, would almost always choose to take any arbitrarily given social security. Second, but if reversely any social security is mandatory, while a fixed intergenerational transfer is not, it may not be the case. If a non-mandatory intergenerational transfer $Y$ coincides with $G$, the maximum utility point, parents are very likely to take both of any arbitrary $K$ and the intergenerational transfer $Y(=G)$, on the other hand, if a non-mandatory intergenerational transfer $Y$ coincides with $F$, the minimum utility point, parents are very likely to take only social security for any arbitrary $K$. This implies that it is quite natural to think that for any arbitrary, but mandatory $K$, which is not extremely large in amount, there exists some point $Y$ on $\underline{X}_{p}$, such that parents are indifferent to whether to accept an intergenerational contract or not. From continuity and monotonicity of parents' indirect utilities on $\underline{X}_{p}$, we have a proposition and a corollary as follows.

Proposition 2: See Figure 2. For any arbitrary social security $K$, which is located on $l_{1}$, such that $O K \leq O^{\prime} Z^{\prime \prime}$, there always exists at least one intergenerational transfer, $\bar{Y}(K)$ as a function of $K$ on $\underline{X}_{p}$, such that $V^{p}(K)=V^{p}(K+\bar{Y}(K))$.

Corollary 2: Assume $\bar{Y}(K)$ is not point $G$. Then $\bar{Y}(K)$ moves slightly along $\underline{X}_{p}(G F)$ in the direction to $G$, for a slight positive change in $K$.

Just for purely mathematical interest, we claim following two lemmas.

Lemma 1: $\underline{X}_{p}$ (A segment line $G F$ ) has a negative tangent slope (of $R$ with regard to 
$P$ ), which is less than -1. Also, a segment line $J D$, which is a part of $\underline{X}_{a c}$, has a negative tangent slope (of $R$ with regard to $P$ ), which is less than -1 .

Lemma 2: Denote a tangent point of parents' indifference curve with a constant premium line, $l_{1}{ }^{\prime}=(1-\varphi) P-\varphi R=k$, by point $E^{k}$. (So, $E^{k=0}$ is the same point as $E$.) Then the locus of the set of point $E^{k}$, has a negative tangent slope (of $R$ with regard to $P$ ), which is less than -1 .

The proof of next Proposition is directly derived from Lemma 2.

Proposition 3: Assume an already concluded (mandatory) intergenerational transfer $Y$ on $\underline{X}_{p}$, and a flexible, actuarially fair social security $K$ on $l_{1}$. Then the optimal social security $\hat{K}(Y)$ as a function of $Y$, which gives the maximum of parents' indirect utility $V^{p}(\hat{K}(Y)+Y)$, decreases in its size $O \hat{K}$, as $Y$ moves along $\underline{X}_{p}$ from $G$ to $F$.

Lastly we examine the simplest case in which only actuarially fair social security $K$ on $l_{1}$ is available for parents. Assume that only actuarially fair social security $K$ on $l_{1}$ is available for parents. Then, as $K$ moves along $l_{1}$ from $O$ to $Q$, that is, as $\tilde{K}$ moves along $\underline{X}_{a c}$ (DJEQ), parents' marginal utility of social security decreases. Especially at point $J$ the marginal utility discontinuously jumps into a lower level, and it becomes 0 (zero) at point $E$. This aspect shows that if social security is some point between $O$ and $J$, the marginal utility (benefit) of social security is relatively high because of the decreasing cost of self-insurance. Together with intergenerational transfer, however, this kind of discontinuity does not appear.

\section{Other results regarding the sensitivity to default risk and taxation in social security}


In this section we limit our analysis only on social security, and examine the sensitivity both of parents and children to default risk and taxation on the demand for social security, wherein now children's income risk $s_{2}$ arises and $W_{1}^{c}>W_{0}^{c} .19$

\section{Sensitivity to default risk}

We introduce another risk $s_{3}$ for the default risk of social security system, where $s_{3}=1$ represents non-default and $s_{3}=0$ represents default. Also assume that the probability of default $\left(s_{3}=0\right)$ is $\eta$, and $s_{3}$ has no correlation with $s_{1}$ and $s_{2}$, respectively. ${ }^{20}$ At first, consider the demand for social security by parents during stage $O$, $\left(R^{p}, P^{p}\right)(=(R, P))$. The pay off of parents for each realization of two relevant risks, $s_{1}$ and $s_{3}$, is as following. Parents receive $R$ for $\left\{s_{1}=1, s_{3}=1\right\}$ with probability $\varphi(1-\eta)$, $-P$ for $\left\{s_{1}=0, s_{3}=1\right\}$ with $(1-\varphi)(1-\eta), 0($ zero $)$ for $\left\{s_{1}=1, s_{3}=0\right\}$ with $\varphi \eta,-P$ for $\left\{s_{1}=0, s_{3}=0\right\}$ with $(1-\varphi) \eta$, respectively. In case of "default", parents still have a liability $(P)$, if they die (that is, if $\left\{s_{1}=0, s_{3}=0\right\}$ occurs). Now we have two definitions for actuarially fair condition: Conditional actuarially fair condition on non-default, $l_{1}: \varphi R=(1-\varphi) P \quad(5.1), \quad$ and $\quad$ unconditional actuarially fair condition, $l_{1}^{\eta}: \varphi(1-\eta) R=(1-\varphi) P(5.2)$. Furthermore, parents' associated indirect utility including default risk is re-defined as:21

$$
\widetilde{\widetilde{v}}^{p}\left(R, P, \varphi, \eta, \beta, r, A^{p}\right) \equiv u\left(A^{p}-P\right)+\varphi(1-\eta) \beta u((1+r)(R+P))+\varphi \eta \beta u((1+r) P)
$$

Now we examine the sensitivity of parents' demand for an actuarially fair social security in the sense of (5.1) and (5.2), when $\eta$ deviates slightly from 0 (zero) by a positive bit. In particular, our interest is in the sensitivity of an optimal contract $E$ and a reservation

19 Therefore condition (iv) of (2.4) has been relaxed.

20 It seems appropriate to assume that there exists no correlation among $s_{1}, s_{2}$ and $s_{3}$, so far as there does not occur any strong social systemic risk. Otherwise, these three risks may have a considerable strong positive correlation with each other.

21 (5.3) is a modified version of (2.4a). 
contract $I$, to $\eta$. In order to do this, we denote the tangent point of either $l_{1}$ or $l_{1}^{\eta}$ with the indifference curve based on this "modified" associated indirect utility (5.3), by $E^{\eta}:\left(R_{E}(\eta), P_{E}(\eta)\right) .22$ Also, we denote the point on either $l_{1}$ or $l_{1}^{\eta}$, which, with the indifference curve based on a modified associated indirect utility, (5.3), attains the same utility as at point $D$ of $\eta=0\left(D^{\eta=0}\right.$, say), by $I^{\eta}:\left(R_{I}(\eta), P_{I}(\eta)\right) .{ }^{23}$ Now we claim a following proposition.

Proposition 4: (Parents' demand sensitivity to default risk) Assume that social security has default risk with conditional actuarially fair condition (5.1). Then we have: (i) $R_{E}{ }^{\prime}(\eta)>0, P_{E}{ }^{\prime}(\eta)>0 \quad$ if $\quad \sigma>1 \quad, \quad R_{E}{ }^{\prime}(\eta)=0, P_{E}{ }^{\prime}(\eta)=0 \quad$ if $\quad \sigma=1 \quad, \quad$ and $R_{E}{ }^{\prime}(\eta)<0, P_{E}{ }^{\prime}(\eta)<0$ if $\sigma<1$. Furthermore we have: (ii) $R_{I}{ }^{\prime}(0)>0, P_{I}{ }^{\prime}(0)>0$, irrespective of the value of $\sigma$.

Instead of (5.1), assume that social security has default risk with unconditional actuarially fair condition (5.2). Then it always holds that: (iii) $R_{E}{ }^{\prime}(\eta)>0, P_{E}{ }^{\prime}(\eta)>0$, and (iv) $R_{I}{ }^{\prime}(0)>0, P_{I}{ }^{\prime}(0)>0$, irrespective of the value of $\sigma$.

Next consider the optimal demand for social security by children during stage $M$, $\left(R^{c}, P^{c}\right)(=(R, P))$. The pay off of children for each realization of two relevant risks, $s_{2}$ and $s_{3}$, is as following. Children receive $-P$ for $\left(s_{2}=1, s_{3}=1\right)$ with probability $\varphi^{\prime}(1-\eta)$, $R$ for $\left\{s_{2}=0, s_{3}=1\right\}$ with $\left(1-\varphi^{\prime}\right)(1-\eta),-P$ for $\left\{s_{2}=1, s_{3}=0\right\}$ with $\varphi^{\prime} \eta, 0$ (zero) for $\left\{s_{2}=0, s_{3}=0\right\}$ with $\left(1-\varphi^{\prime}\right) \eta$, respectively. In case of "default", children still have a liability $(P)$, if they have a higher income (that is, if $\left\{s_{2}=1, s_{3}=0\right\}$ occurs). Now we have two definitions for actuarially fair condition: Conditional actuarially fair condition on

${ }^{22} E^{\eta=0}:\left(R_{E}(0), P_{E}(0)\right)$ coincides with point $E:\left(S_{E}, B_{E}\right)$.

${ }_{23} I^{\eta}$ is the point, which, with the default risk, assures the same minimum utility as when no social security is available. $I^{\eta=0}:\left(R_{I}(0), P_{I}(0)\right)$ coincides with point $I:\left(S_{I}, B_{I}\right)$. 
non-default, $l_{3}: \varphi^{\prime} P=\left(1-\varphi^{\prime}\right) R \quad(5.4)$, and unconditional actuarially fair condition, $l_{3}^{\eta}: \varphi^{\prime} P=\left(1-\varphi^{\prime}\right)(1-\eta) R \quad$ (5.5). Furthermore, children's "modified" associated indirect utility including default risk is defined as: 24

$$
\widetilde{\widetilde{v}}^{c}\left(P, R, \varphi^{\prime}, \beta, \eta, r, W^{c}\right) \equiv \max _{c_{1}}\left(\begin{array}{l}
\varphi^{\prime}\left[u\left(c_{1}\right)+\beta u\left((1+r)\left(W_{1}^{c}-P-c_{1}\right)\right)\right] \\
+\left(1-\varphi^{\prime}\right)(1-\eta)\left[u\left(c_{1}\right)+\beta u\left((1+r)\left(W_{0}^{c}+R-c_{1}\right)\right)\right] \\
+\left(1-\varphi^{\prime}\right) \eta\left[u\left(c_{1}\right)+\beta u\left((1+r)\left(W_{0}^{c}-c_{1}\right)\right)\right]
\end{array}\right)
$$

Now we examine the sensitivity of children's optimal demand for an actuarially fair social security $\left(R^{c}(\eta), P^{c}(\eta)\right)$ under (5.4) and (5.5) to $\eta$, when $\eta$ deviates slightly from 0 by a positive bit. Then:

Proposition 5: (Children's demand sensitivity to default risk) Assume that social security has default risk with conditional actuarially fair condition (5.4). Then (i) $R^{c^{\prime}}(0)<0, P^{c^{\prime}}(0)<0$. Instead of (5.4), assume that social security has default risk with unconditional actuarially fair condition (5.5). Then (ii) $R^{c \prime}(0)>0, P^{c \prime}(0)<0$.

In either case of (5.4) or (5.5), $R^{c}(0)=\varphi^{\prime}\left(W_{1}^{c}-W_{0}^{c}\right)$ and $P^{c}(0)=\left(1-\varphi^{\prime}\right)\left(W_{1}^{c}-W_{0}^{c}\right)$, where children fully insure their income risk.

Sensitivity to taxation

We turn our focus to taxation on social security both for parents and children. Consider two kinds of tax: a lump-sum actuarially fair tax, and an exercise tax only on payment $P$. Let $T_{R}$ and $T_{P}$ be conditional taxes imposed on the realization of receipt $R$ and payment $P$, respectively. Lump-sum actuarially fair tax is described as $\left(T_{R}, T_{P}\right)$, where $\varphi T_{R}=(1-\varphi) T_{P}$ for parents and $\left(1-\varphi^{\prime}\right) T_{R}=\varphi^{\prime} T_{P}$ for children, (5.7). An exercise tax on

24 (5.6) is a modified version of $(2.4 \mathrm{~b})$. 
payment $P$, is described as $\left(T_{R}, T_{P}\right)=\left(0, T_{p}\right)$, where $T_{P}=\zeta P$, and we assume tax-deducted actuarially fair conditions, $\varphi R=(1-\varphi)(1-\zeta) P \quad(5.8)$ for parents, $\left(1-\varphi^{\prime}\right) R=\varphi^{\prime}(1-\zeta) P(5.9)$ for children. $\zeta$ is defined as a proportional tax rate on $P$. The expected tax income by the government is, $E T \equiv(1-\varphi) T_{P}-\varphi T_{R}$ (5.10) for parents, and $E T \equiv \varphi^{\prime} T_{P}-\left(1-\varphi^{\prime}\right) T_{R}$ (5.11) for children. The associated indirect utilities with regard to $(R, P)$, remain almost the same as $(2.4 \mathrm{a})$ for parents, and $(2.4 \mathrm{~b})$ for children:

$$
\begin{aligned}
& \widetilde{v}^{p}\left(R, P, \varphi, \beta, r, A^{p}\right) \equiv u\left(A^{p}-P\right)+\varphi \beta u((1+r)(R+P)) \\
& \widetilde{v}^{c}\left(P, R, \varphi, \beta, r, \bar{W}^{c}\right) \equiv \max _{c_{1}}\left(\begin{array}{l}
\varphi^{\prime}\left[u\left(c_{1}\right)+\beta u\left((1+r)\left(\bar{W}_{1}^{c}-P-c_{1}\right)\right)\right] \\
+\left(1-\varphi^{\prime}\right)\left[u\left(c_{1}\right)+\beta u\left((1+r)\left(\bar{W}_{0}^{c}+R-c_{1}\right)\right)\right]
\end{array}\right)
\end{aligned}
$$

Clearly a lump-sum actuarially fair tax is better than an exercise tax on payment $P$, for both parents and children, in the sense that, keeping the expected tax income ET at constant, a lump-sum actuarially fair tax could always attain better associated indirect utility with regard to $(R, P)$, than an exercise tax only on payment $P$.

We proceed to the sensitivity analysis to an exercise taxation on payment $P$, as described in (5.8) for parents and (5.9) for children. (5.8) and (5.9) are, in a sense, equivalent with unconditional actuarially fair conditions incorporating default risk, (5.2) for parents and (5.5) for children, respectively, if we set $(1-\eta)(1-\zeta)=1$. Here, we can interpret $\eta$ as a conditional profit margin or a subsidy margin on the realization of receipt $R$. Denote parents' optimal demand for social security with the condition $(5.8)$ by $\left(\widetilde{R}^{p}(\zeta), \widetilde{P}^{p}(\zeta)\right)$, and children's optimal demand for social security with the condition (5.9) by $\left(\widetilde{R}^{c}(\zeta), \widetilde{P}^{c}(\zeta)\right)$, respectively. Then:

Proposition 6: (Parents' demand sensitivity to taxation) Assume that the government imposes an exercise tax on payment $P$, for parents' social security, with tax-deducted 
actuarially fair condition (5.8). Then (i) $\widetilde{R}^{p{ }^{\prime}}(\zeta)<0$, irrespective of the value of $\sigma$. Furthermore (ii) $\widetilde{P}^{p}(\zeta)>0$ if $\sigma>1, \widetilde{P}^{p}(\zeta)=0$ if $\sigma=1$, and $\widetilde{P}^{p^{\prime}}(\zeta)<0$ if $\sigma<1$. Proposition 7: (Children's demand sensitivity to taxation) Assume that the government imposes an exercise tax on payment $P$ for children's social security, with tax-deducted actuarially fair condition (5.9). Then (i) $\widetilde{R}^{c}(0)<0$, irrespective of the value of $\sigma$.

\section{Final remarks}

In a continuous-time case, parents' problem to be solved can be represented as follows: Define parents' transfer contract incorporating the risk of death $(S(t), B(t))$ for $0 \leq t \leq T$. $T$ (亡30 years) is the length of stage $O$, and $(S(t), B(t))$ is measured as a present value at time 0 , not $t$, and $B(t)$ is continuously differentiable for all $0 \leq t \leq T$. Also let $c(t)$ be their consumption plan at time t, measured as a present value at time $t$, and $A^{p}$ be the present value of total available wealth, measured at time 0 . Define the probability that parents are alive at time $\mathrm{t}$, as $\varphi(t)$, where $\varphi(0)=1$, and $\varphi(T)=0$. Then the budget (feasibility) constraint is written as:

$$
\int_{0}^{t}\left\{-S\left(t^{\prime}\right)+c\left(t^{\prime}\right) \exp \left(-r t^{\prime}\right)\right\} d t^{\prime}=A^{p}-B(t) \text { for all } 0 \leq t \leq T
$$

Equivalently in a differential form: $c(t)=\left[-B^{\prime}(t)+S(t)\right] \exp (r t)$ for all $0 \leq t \leq T\left(6.1^{\prime}\right)$

From (6.1), we have $B(0)=A^{p}$, and $B(T)=0$. An actuarially fair condition of the transfer

contract $(S(t), B(t))$ is: $\int_{0}^{T} \varphi(t) S(t) d t=-\int_{0}^{T} B(t) \varphi^{\prime}(t) d t$

Here $\widetilde{S} \equiv \int_{0}^{T} \varphi(t) S(t) d t$ is an expected support and $\widetilde{B} \equiv-\int_{0}^{T} B(t) \varphi^{\prime}(t) d t$ is an expected bequest. In a differential form, $\varphi(t) S(t)=-B(t) \varphi^{\prime}(t)\left(6.2^{\prime}\right)$. This is a continuous-time version of an actuarially fair condition in a two-period case, $l_{1}: \varphi S=(1-\varphi) B$. Assume that 
parents' transfer schedule $(S(t), B(t))$ is predetermined. Then they solve: (X) $\max _{c(t)} \int_{0}^{T} \varphi(t) u(c(t)) \exp (-\beta t) d t \quad$ (6.3) $\quad$ s.t. (6.1'). However, as a matter of fact the maximization problem is not left for parents, but their consumption is automatically determined at $c(t)=\left[-B^{\prime}(t)+S(t)\right] \exp (r t)$ for all $0 \leq t \leq T\left(6.1^{\prime}\right)$. Therefore, as in a two-period case, there exists some possibility of self-insurance, in which parents must pay an additional cost conditionally on death. On the other hand, children's indirect utility at time $t$ with transfer contract $(S(t), B(t))(0 \leq t \leq T)$ proves to have an indifference curve, which is tangent with a continuous-time actuarially fair line $\varphi(t) S(t)=-B(t) \varphi^{\prime}(t) \quad\left(6.2^{\prime}\right)$. From all the above points, our analysis made in the previous 4 sections with two discrete periods does not lose any generality even in a continuous-time case.

Thus this article has just summarized, using a simple model of two discrete periods and binary states of mortality/income risks, some fundamental propositions regarding the mixture of intergenerational risk sharing and social security. Here for the first time, state-dependent utility under mortality risk proves to generate parents' peculiar indifference curve regarding insurance contract, and self-insurance is shown to play a crucial role on the decision regarding social security holding and intergenerational transfer contract.

\section{Reference}

Atkinson, Anthony B. and Joseph E. Stiglitz (1980) "Lecture on Public Economics", McGraw-Hill.

Obstfeld, Maurice and Kenneth Rogoff (1996) "Foundation of International Macroeconomics", The MIT Press

Ehrlich, Isaac and Gary Becker (1972) "Market Insurance, Self-Insurance, and Self-Protection", The Journal of Political Economy, Vol. 80, Issue 4, Jul.-Aug. 1972, 623-648. Ball, Laurence and N. Gregory Mankiw (2001) "Intergenerational Risk Sharing in the Spirit of Arrow, Debreu, and Rawls, with Applications to Social Security Design", NBER Working Paper No.8270, May 2001.

Enders, Walter and Harvey E. Lapan (1982) "Social Security Taxation and Intergenerational Risk Sharing”, International Economic Review, Vol.23, Issue 3, Oct. 1982, 647-658.

Gordon, Roger H. and Hal R. Varian (1988) "Intergenerational Risk Sharing", Journal of Public Economics, Vol. 37, 1988, 185-202. 
Yaari, Menahem E. (1965) "Uncertain Lifetime, Life Insurance, and the Theory of the Consumer", The Review of Economic Studies, Vol. 32, Issue 2, 1965, 137-150.

Aoki, Takaaki (2003) "Essay on the Intergenerational Risk Sharing, Social Security and Taxation", mimeo (Unpublished) (The First Version, Term paper for Eco 743, State University of New York at Buffalo, Dept. of Economics), Jan. 2003., "Self-insurance and Some Aspects on the Intergenerational Risk Sharing and Social Security", mimeo (Unpublished) (The second Version: Sep. 2003, the third Version: Jan. 2004, the last version: June 2005).

Hayashi, Fumio, Joseph Altonji and Laurence Kotlikoff (1996) "Risk-Sharing between and within Families", Econometrica, Vol.64, Issue 2, Mar. 1996, 261-294.

Abel, Andrew B. (1987) "Operative Gift and Bequest Motives", The American Economic Review, Vol. 77, Issue 5, pp. 1037-1047, Dec. 1987.

Abel, Andrew B. (1985) "Precautionary Saving and Accidental Bequest", The American Economic Review, Vol.75, Issue 4, Sep. 1985, 771-791.

Hurd, Michael D. (1989) "Mortality Risk and Bequests", Econometrica, Vol.57, Issue 4, Jul. 1989, 779-813.

Bernheim, Douglas B., Andrei Shleifer and Lawrence H. Summers (1985) "The Strategic Bequest Motive", The Journal of Political Economy, Vol. 93, Issue 6, Dec. 1985, 1045-1076.

Bernheim, Douglas B. (1991) "How Strong Are Bequest Motives? Evidence Based on Estimates of the Demand for Life Insurance and Annuities", The Journal of Political Economy, Vol. 99, Issue 5, Oct. 1991, 899-927.

Drazen, Allan (1978) "Government Debt, Human Capital, and Bequests in a Life-Cycle Model", The Journal of Political Economy, Vol.86, Issue 3, Jun. 1978, 505-516.

Shiller, Robert J. (1998) "Social Security and Institutions for Intergenerational, Intragenerational and International Risk Sharing", Carnegie-Rochester Series in Public Policy, Vol. 50: 165-205, June 1999. 


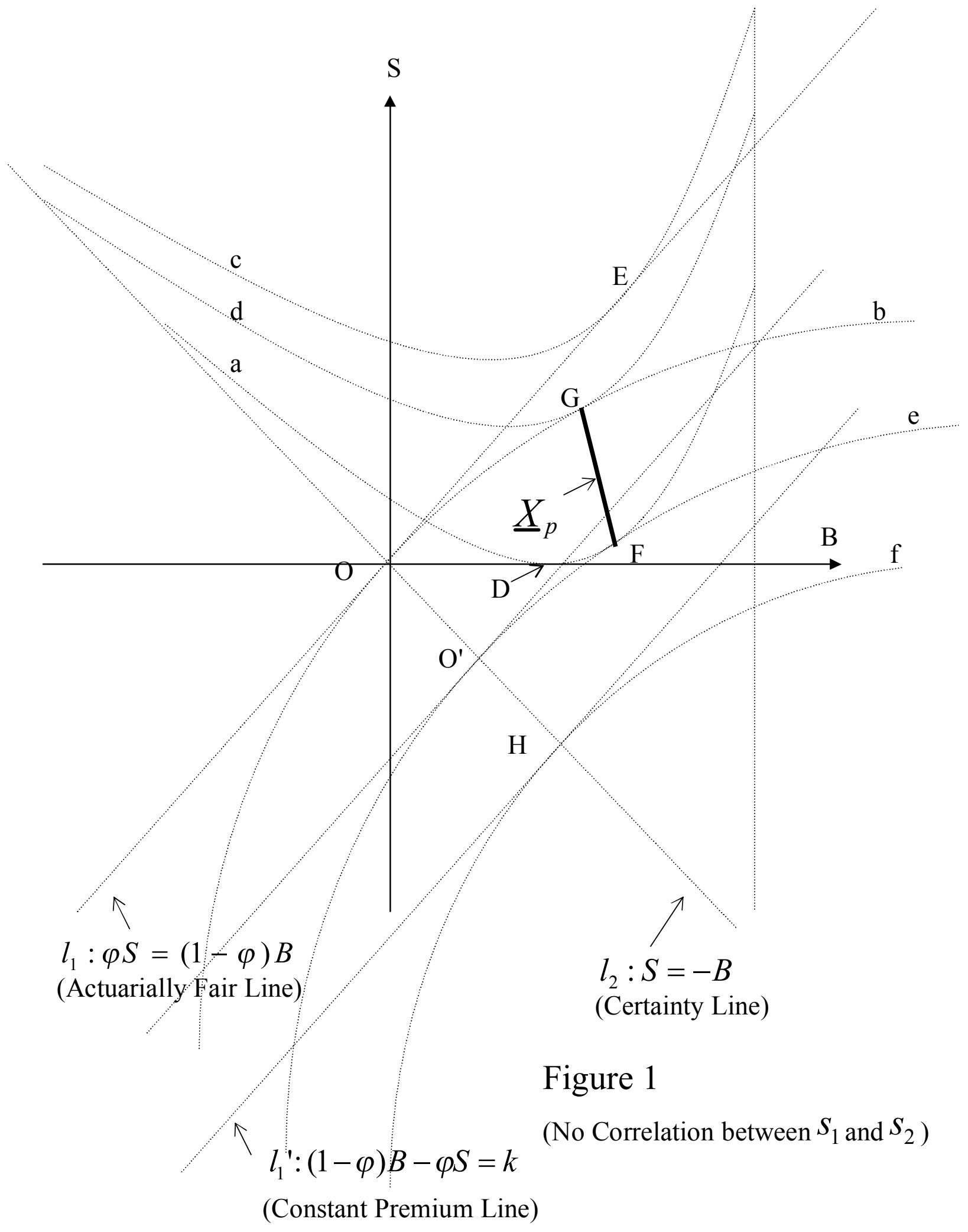




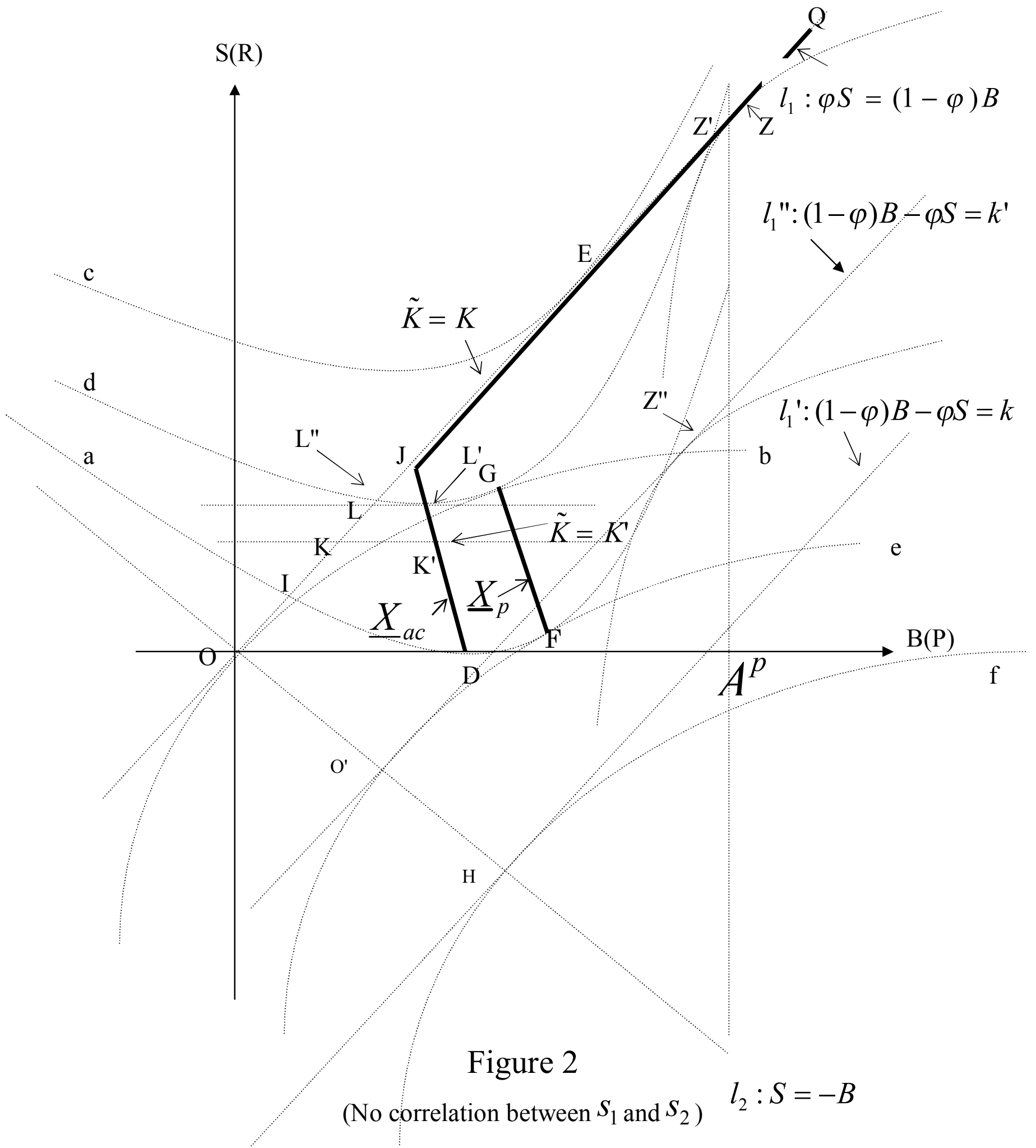




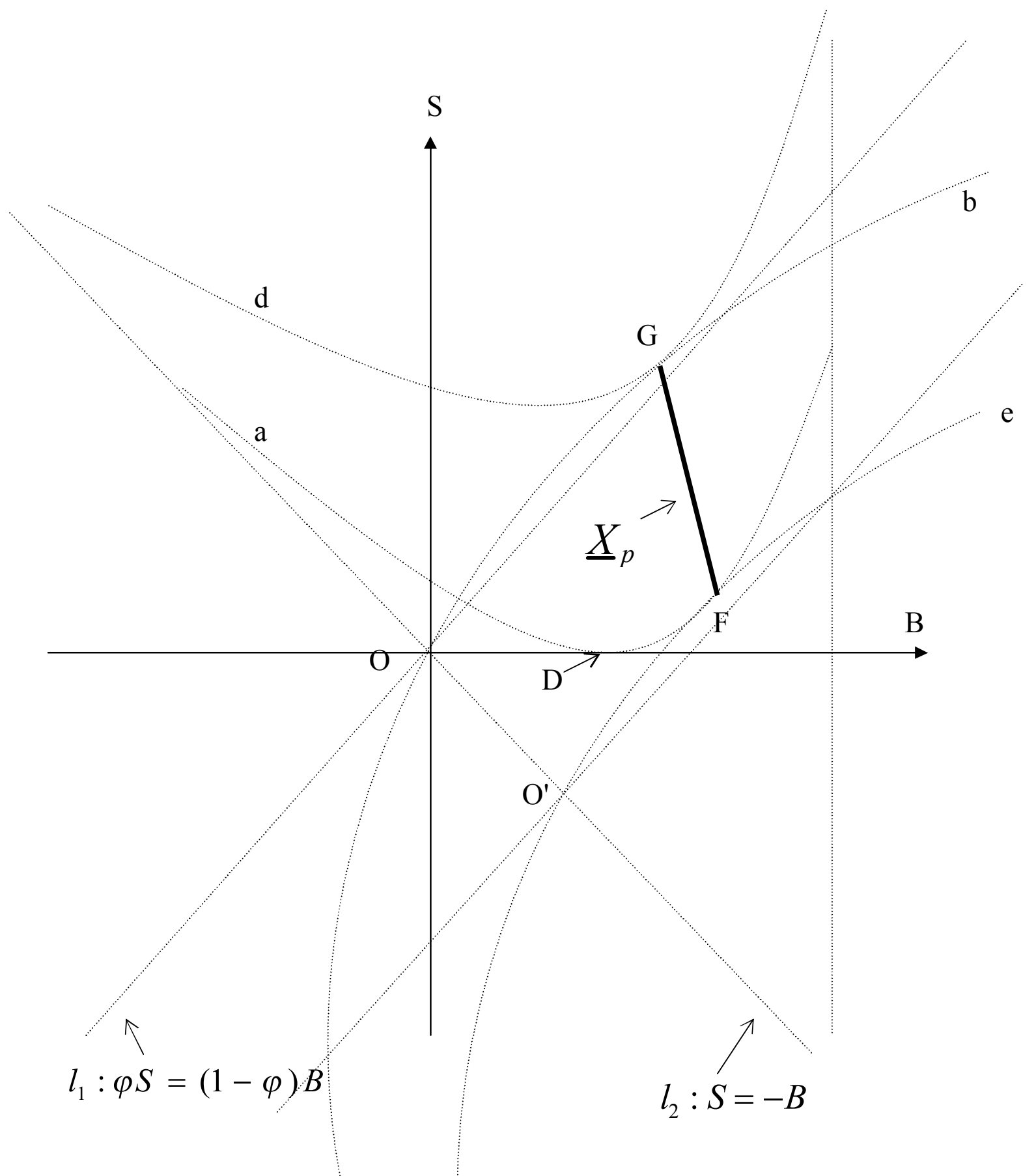

Figure 3

(Positive Correlation between $S_{1}$ and $S_{2}$ ) 


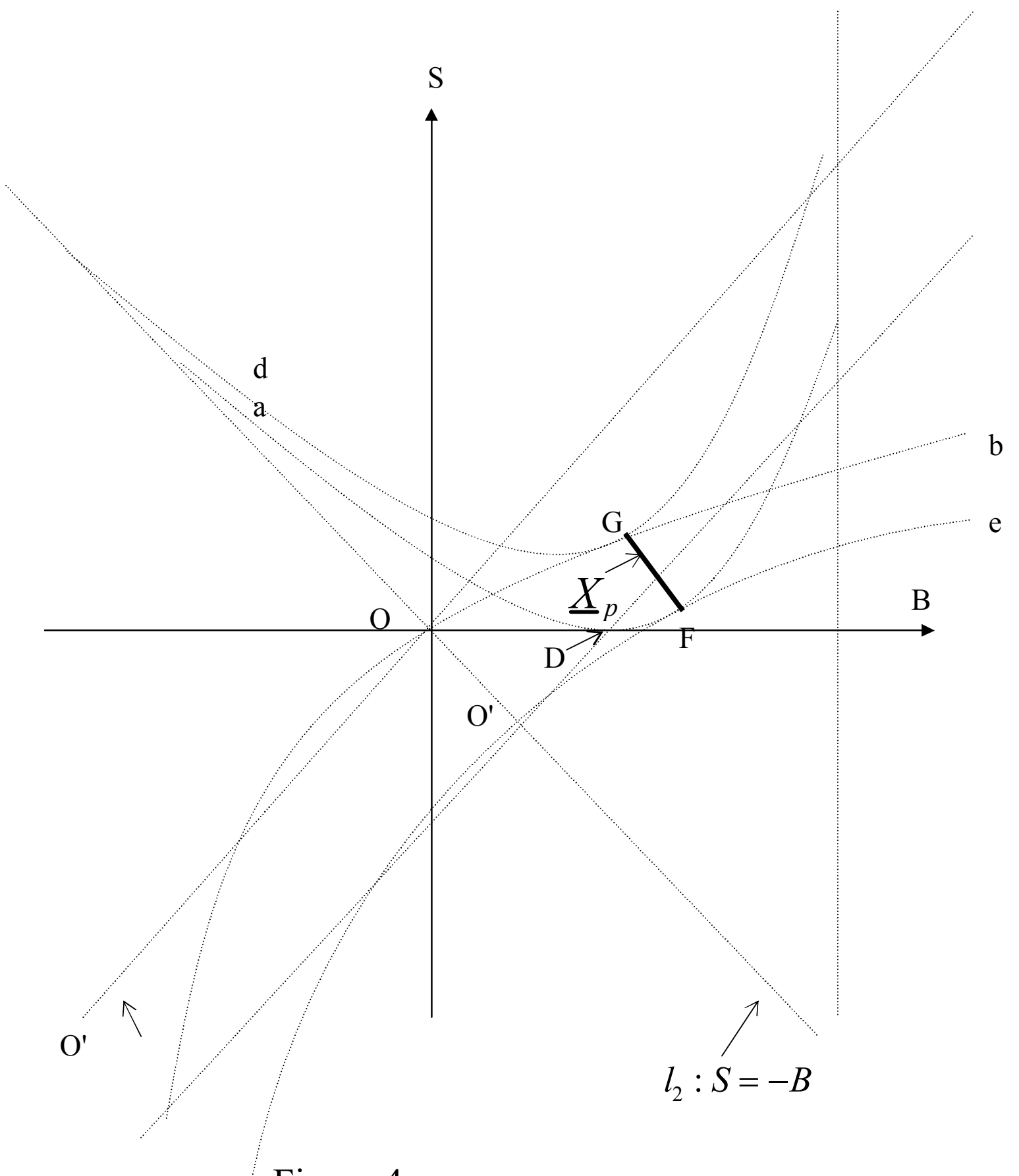

Figure 4

(Negative Correlation between $S_{1}$ and $S_{2}$ ) 


\section{Appendix}

Rigorous definitions of $V^{P}(K), V^{P}(Y)$ and $V^{P}(K+Y)$

Define an actuarially fair social security $K:\left(R_{K}, P_{K}\right)$, where $K$ is on $l_{1}: \varphi R_{K}=(1-\varphi) P_{K}$, and an intergenerational transfer $Y:\left(S_{Y}, B_{Y}\right)$ on $\underline{X}_{P}$. Then:

$$
\begin{aligned}
& V^{p}(K) \equiv \max _{P^{\prime} \geq 0} \widetilde{v}^{p}\left(R_{K}, P_{K}+P^{\prime}, \varphi, \beta, r, A^{p}\right) \\
& V^{p}(Y) \equiv \widetilde{v}^{p}\left(S_{Y}, B_{Y}, \varphi, \beta, r, A^{p}\right) \\
& V^{p}(K+Y) \equiv \max _{(S, B), P^{\prime} \geq 0} \widetilde{v}^{p}\left(R_{K}+S, P_{K}+B+P^{\prime}, \varphi, \beta, r, A^{p}\right) \\
& \text { s.t. } \tilde{v}^{c}\left(S, B, \varphi, \beta, r, \bar{W}^{c}\right)=\widetilde{v}^{c}\left(S_{Y}, B_{Y}, \varphi, \beta, r, \bar{W}^{c}\right)\left(\equiv \bar{v}^{c}\right) \\
& \text { and } l_{1}: \varphi R_{K}=(1-\varphi) P_{K}
\end{aligned}
$$

\section{Proof of Proposition 1:}

See Figure 2. Graphically $V^{P}(K)$ can be determined as parents' indirect utility of the point, where a horizontal line $R=R_{K}$, crosses $\underline{X}_{a c}$ (that is, parents' indifference curve is tangent with a horizontal line $R=R_{K}$ clearly on $\left.\underline{X}_{a c}\right), V^{P}(Y)$ simply as that of point $Y$ on $\underline{X}_{P}$, and $V^{P}(K+Y)$ as that of the point where parents' indifference curve is tangent with the curve generated by shifting children's indifference curve in parallel along with $l_{1}$ by vector $\left(R_{K}, P_{K}\right)$.

(i) (Case: $Y=G$ ) Let $Z$ be the point where children's "shifted" indifference curve, which is tangent both with parents' indifference curve $d$, and an actuarially fair line $l_{1}$, is tangent

$25(\mathrm{~A} 1.1-1)$ can be rewritten as:

$$
V^{p}(K) \equiv \max _{R=R_{K}, P \geq P_{K}} \tilde{v}^{p}\left(R, P, \varphi, \beta, r, A^{p}\right)
$$

26 (A1.1-3) can be rewritten as:

$$
\begin{aligned}
& V^{p}(K+Y) \equiv \max _{(R, P), P^{\prime} \geq 0} \tilde{v}^{p}\left(R, P+P^{\prime}, \varphi, \beta, r, A^{p}\right) \\
& \text { s.t. } \widetilde{v}^{c}\left(R-R_{K}, P-P_{K}, \varphi, \beta, r, \bar{W}^{c}\right)=\widetilde{v}^{c}\left(S_{Y}, B_{Y}, \varphi, \beta, r, \bar{W}^{c}\right)\left(\equiv \bar{v}^{c}\right) \\
& \quad \text { and } l_{1}: \varphi R_{K}=(1-\varphi) P_{K}
\end{aligned}
$$


with $l_{1}$. For some social security $K$, children's shifted indifference curve is denoted by $b^{\prime}$ for the original indifference curve $b$. Graphically it is clear that for any arbitrary $K$ on $l_{1}$ such that $O K \leq O Z$, parents' indifference curve, which is tangent with children's shifted indifference curve $b^{\prime}$, is located in the upper side of both point $K$ and indifference curve $d$ (or point $G$ ). So, we have $V^{p}(Y(=G)) \leq V^{p}(K+Y(=G))$ with equality when $K=Z$, and $V^{p}(K) \leq V^{p}(K+Y(=G))$ with equality when $K=E$.

(ii) (Case: $Y=F$ ) Denote the point where children's indifference curve $e$ crosses the certainty line $l_{2}: R=-P$, by $O^{\prime}$. Also denote some constant premium line, which passes $O^{\prime}$, by $l_{1}$ ": $(1-\varphi) P-\varphi R=k^{\prime}$. (So, $e$ is tangent with parents' indifference curve $a$ at point $F$, and also is tangent with $l_{1}^{\prime \prime}$ at point $\left.O^{\prime}.\right) Z^{\prime \prime}$ is the point where children's shifted indifference curve, which is tangent with both parents' indifference curve $a$ and a constant premium line $l_{1}^{\prime \prime}$, is tangent with $l_{1}^{\prime \prime}$. For some social security $K$, denote children's shifted indifference curve, which corresponds with original indifference curve $e$, by $e^{\prime}$. Graphically it is clear that for any arbitrary $K$ on $l_{1}$, such that $O K \leq O^{\prime} Z^{\prime \prime}$, that parents' indifference curve, which is tangent with children's shifted indifference curve $e^{\prime}$, is located in the upper side of parents' indifference curve $a$ (or point $F$ ). So we have $V^{p}(Y(=F)) \leq V^{p}(K+Y(=F))$ with equality when $O K=O^{\prime} Z^{\prime \prime}$. Now we proceed to the proof of $V^{p}(K) \geq V^{p}(K+Y(=F))$. For later convenience, we rewrite $V^{p}(K)$ and $V^{p}(K+Y)$ as $V^{p}\left(R_{K}\right)$ and $V^{p}\left(R_{K}, Y\right)$ using the amount of receipt for social security $K, R_{K}$. Clearly, when $K=O$ (zero receipt, zero payment), we have $V^{p}\left(R_{K}(=0)\right)=V^{p}\left(R_{K}(=0), Y(=F)\right),{ }^{27}$ because parents are indifferent between $D$ and $F$. So, at first we show that,

${ }^{27}$ That is, $V^{p}(K(=O))=V^{p}(K(=O)+Y(=F))$. 


$$
\frac{\partial}{\partial R_{K}} V^{p}\left(R_{K}(=0)\right)>\frac{\partial}{\partial R_{K}} V^{p}\left(R_{K}(=0), Y(=F)\right),
$$

and next show that $V^{p}\left(R_{K}, Y(=F)\right)$ can never catch and overpass $V^{p}\left(R_{K}\right)$ for any arbitrary $K$ such that $O K \leq O^{\prime} Z^{\prime \prime}$.

First step: Since, given an intergenerational transfer $Y$, parents need not pay an additional cost on death (i.e., one constraint $P^{\prime} \geq 0$ is binding.), we can rewrite (A1.1-3) as:

$$
\begin{aligned}
& V^{p}\left(R_{K}, Y\right) \equiv \max _{(R, P)} \widetilde{v}^{p}(R, P, \cdots) \\
& \text { s.t. } \widetilde{v}^{c}\left(R-R_{K}, P-P_{K}, \cdots\right)=\widetilde{v}^{c}\left(S_{Y}, B_{Y}, \cdots\right)\left(\equiv \bar{v}^{c}\right) \\
& \quad \text { and } l_{1}: \varphi R_{K}=(1-\varphi) P_{K}
\end{aligned}
$$

Here $(R, P)$ denotes a "mixed" transfer contract schedule. Plugging the second equation of constraints into other equations of (A1.3), we have a following Lagrangian and corresponding first order conditions.

$$
\begin{gathered}
L=\widetilde{v}^{p}(R, P, \cdots)-\lambda\left(\widetilde{v}^{c}\left(R-R_{K}, P-\frac{\varphi}{1-\varphi} R_{K}, \cdots\right)-\bar{v}^{c}\right) \\
\frac{\partial}{\partial R} \widetilde{v}^{p}(R, P, \cdots)-\lambda \frac{\partial}{\partial R} \widetilde{v}^{c}\left(R-R_{K}, P-\frac{\varphi}{1-\varphi} R_{K}, \cdots\right)=0 \\
\frac{\partial}{\partial P} \widetilde{v}^{p}(R, P, \cdots)-\lambda \frac{\partial}{\partial P} \widetilde{v}^{c}\left(R-R_{K}, P-\frac{\varphi}{1-\varphi} R_{K}, \cdots\right)=0
\end{gathered}
$$

From the envelope theorem, also using (A1.3), we obtain:

$$
\begin{aligned}
& V^{p}\left(R_{K}, Y\right) \equiv \frac{\partial}{\partial R_{K}} V^{p}\left(R_{K}, Y\right) \\
& =\lambda\left(\frac{\partial}{\partial R} \widetilde{v}^{c}\left(R-R_{K}, P-\frac{\varphi}{1-\varphi} R_{K}, \cdots\right)+\frac{\varphi}{1-\varphi} \frac{\partial}{\partial P} \widetilde{v}^{c}\left(R-R_{K}, P-\frac{\varphi}{1-\varphi} R_{K}, \cdots\right)\right) \\
& =\frac{\partial}{\partial R} \widetilde{v}^{P}(R, P, \cdots)+\frac{\varphi}{1-\varphi} \frac{\partial}{\partial P} \widetilde{v}^{P}(R, P, \cdots) \equiv \Xi(R, P)
\end{aligned}
$$

Similarly we obtain the following equation quite easily:

$$
\begin{aligned}
& V^{p}{ }^{\prime}\left(R_{K}\right) \equiv \frac{\partial}{\partial R_{K}} V^{p}\left(R_{K}\right) \\
& =\frac{\partial}{\partial R} \widetilde{v}^{P}(R, P, \cdots)
\end{aligned}
$$


Denote the point at which parents indifference curve, which crosses a solution point of (A1.3), $\Omega:\left(R_{\Omega}, P_{\Omega}\right)$ say, intersects with $\underline{X}_{a c}$, by $\Omega_{a c}:\left(R_{\Omega_{a c}}, P_{\Omega_{a c}}\right)$. Clearly, at any arbitrary point on $\underline{X}_{a c}$, we have $\frac{\partial}{\partial P} \widetilde{v}^{P}(R, P, \cdots)=0$, so (A1.5) and (A1.6) actually share the same value at $\Omega$ and $\Omega_{a c}$, that is $\Xi(R, P)$. Now we have only to show that $\Xi(R, P)$ is decreasing as $P$ increases (moves) along parents' indifference curve from $\Omega_{a c}$ to $\Omega$.

Here, denote parents' indifference curve, which passes $\Omega_{a c}$ and $\Omega$, by $g$. Remember parents' indirect utility:

$$
\widetilde{v}^{p}\left(R, P, \varphi, \beta, r, A^{p}\right) \equiv u\left(A^{p}-P\right)+\varphi \beta u((1+r)(R+P))
$$

It follows directly that:28

$$
\begin{aligned}
& \frac{\partial^{2}}{\partial R^{2}} \widetilde{v}^{P}(R, P, \cdots)=\frac{\partial^{2}}{\partial P \partial R} \widetilde{v}^{P}(R, P, \cdots)<0 \\
& \frac{\partial^{2}}{\partial P^{2}} \widetilde{v}^{P}(R, P, \cdots)<0
\end{aligned}
$$

From the definition, we also have;

$$
\Xi(R, P) \equiv \frac{\partial}{\partial R} \widetilde{v}^{P}(R, P, \cdots)+\frac{\varphi}{1-\varphi} \frac{\partial}{\partial P} \widetilde{v}^{P}(R, P, \cdots)
$$

So, from (A1.7) and (A1.8), it follows that:

$$
\begin{aligned}
& \frac{\partial}{\partial R} \Xi(R, P) \equiv \frac{\partial^{2}}{\partial R^{2}} \widetilde{v}^{P}(R, P, \cdots)+\frac{\varphi}{1-\varphi} \frac{\partial^{2}}{\partial P \partial R} \widetilde{v}^{P}(R, P, \cdots)<0 \\
& \frac{\partial}{\partial P} \Xi(R, P) \equiv \frac{\partial^{2}}{\partial P \partial R} \widetilde{v}^{P}(R, P, \cdots)+\frac{\varphi}{1-\varphi} \frac{\partial^{2}}{\partial P^{2}} \widetilde{v}^{P}(R, P, \cdots)<0 \\
& \left.\frac{d R}{d P}\right|_{*}>0 \text { (As graphically clear, } P \text { increases (moves) along parents indifference curve }
\end{aligned}
$$

${ }^{28}$ For the first order condition, we have $\frac{\partial}{\partial R} \widetilde{v}^{P}(R, P, \cdots)>0$. Furthermore, if $P$ is located in the right side of $D J$ on $\left.\underline{X}_{a c}\right)$, then $\frac{\partial}{\partial P} \widetilde{v}^{P}(R, P, \cdots)<0$. 
where * denotes that $(R, P)$ is on $g: \widetilde{v}^{p}(R, P, \cdots)=\bar{v}_{\Omega, \Omega_{a c}}^{p}$, say.

Now, since $\left.\frac{d}{d P} \Xi(R, P)\right|_{*}=\left.\frac{\partial}{\partial R} \Xi(R, P) \frac{d R}{d P}\right|_{*}+\frac{\partial}{\partial P} \Xi(R, P)$,

and also $d P>0$, from (A1.9) and (A1.10) we get $\left.\frac{d}{d P} \Xi(R, P)\right|_{*}<0$ (A1.11). If $\Omega=F$, clearly $\Omega_{a c}=D$, so we have completed the first step, that is, have proved (A1.2).

Second step: Assume that for some $K:\left(R_{K}, P_{K}\right)$, we have $V^{p}(K)=V^{p}(K+Y(=F))$, in another expression, $V^{p}\left(R_{K}\right)=V^{p}\left(R_{K}, Y(=F)\right)$. For this $K$, denote solution points (of mixed contract schedule $(R, P))$ for $\left(\mathrm{A} 1.1-3^{\prime}\right)$ and (A1.3), by $\Omega$ again, and $\Sigma:\left(R_{\Sigma}, P_{\Sigma}\right)$, respectively. $\Omega$ and $\Sigma$ attain the same indirect utility for parents, so $\Sigma$ should coincide with $\Omega_{a c}$ in the above notation. Then we can use the same inequality (A1.11), in order to prove:

$$
\frac{\partial}{\partial R_{K}} V^{p}\left(R_{K}\left(=R_{\Sigma}=R_{\Omega_{a c}}\right)\right)>\frac{\partial}{\partial R_{K}} V^{p}\left(R_{K}\left(=R_{\Omega}\right), Y(=F)\right)
$$

From the continuity of $V^{p}\left(R_{K}\right)$ and $V^{p}\left(R_{K}, Y(=F)\right)$ with regard to $R_{K}$, now we have just proved $V^{p}\left(R_{K}\right) \geq V^{p}\left(R_{K}, Y(=F)\right)$, that is, $V^{p}(K) \geq V^{p}(K+Y(=F))$ for any arbitrary $K$ such that $O K \leq O^{\prime} Z^{\prime \prime}$.

\section{Proof of Lemma 1:}

Graphically it is clear that, for any points on a segment line $J D$ of $\underline{X}_{a c}$, which is the subset of solution points for (A1.1-1), one constraint $P^{\prime} \geq 0$ is not binding. So, from the first order condition, we have a following equality:

$$
\frac{\partial \tilde{v}^{p}}{\partial P}=-u^{\prime}\left(A^{p}-P\right)+\varphi \beta(1+r) u^{\prime}\left((1+r)\left(R_{K}+P\right)\right)=0
$$

Taking $R$ as a function of $P$, and differentiate (A2.1) with regard to $P$, we obtain:

$$
u^{\prime \prime}\left(A^{p}-P\right)+\varphi \beta(1+r)^{2} u^{\prime \prime}\left((1+r)\left(R_{K}+P\right)\right)\left(\frac{d R_{K}}{d P}+1\right)=0
$$


Considering $u^{\prime \prime}<0$, it follows directly that $\frac{d R_{K}}{d P}<-1$ (A2.3).

\section{Proof of Lemma 2:}

Plugging the constant premium condition $l_{1}{ }^{\prime}=(1-\varphi) P-\varphi R=k \quad$ (A2.4) into (2.4a), we obtain the first order condition:

$$
-u^{\prime}\left(A^{p}-P\right)+\beta(1+r) u^{\prime}\left((1+r)\left(\frac{P-k}{\varphi}\right)\right)=0
$$

Taking $P$ as a function of $k$, and differentiate (A2.4) with regard to $k$, we obtain;

$$
u^{\prime \prime}\left(A^{p}-P\right) \frac{d P}{d k}+\frac{\beta(1+r)^{2}}{\varphi} u^{\prime \prime}\left((1+r)\left(\frac{P-k}{\varphi}\right)\right)\left(\frac{d P}{d k}-1\right)=0
$$

from which it follows that $0<\frac{d P}{d k}<1$ or $\frac{d k}{d P}>1$. Since, from (A2.4);

$$
\frac{d R}{d P}=\frac{1-\varphi}{\varphi}-\frac{1}{\varphi} \frac{d k}{d P}
$$

we have $\frac{d R}{d P}<-1$. Now the proof is done.

\section{Proof of Proposition 4:}

Proof of (i): Parents' associated indirect utility including default risk is given in (5.3). Maximizing (5.3) subject to (5.1) with regard to $P$ (and implicitly $R$ ), we have the first order condition for point $E^{\eta}$ :

$$
-u^{\prime}\left(A^{p}-P\right)+(1-\eta) \beta(1+r) u^{\prime}((1+r) P / \varphi)+\varphi \eta \beta(1+r) u^{\prime}((1+r) P)=0 \quad(\mathrm{~A} 3.1)
$$

Replacing $P$ with $P(\eta)$ as a function of $\eta$ in (A3.1), differentiating the equality with regard to $\eta$, and implementing the comparative statics immediately produces the following equation: 


$$
\begin{aligned}
& P^{\prime}(\eta)\left\{u^{\prime \prime}\left(A^{p}-P(\eta)\right)+\left[(1-\eta) \beta(1+r)^{2} / \varphi\right] u^{\prime \prime}((1+r) P(\eta) / \varphi)\right. \\
& \left.+\varphi \eta \beta(1+r)^{2} u^{\prime \prime}((1+r) P(\eta))\right\} \\
& =\beta(1+r)\left[u^{\prime}((1+r) P(\eta) / \varphi)-\varphi u^{\prime}((1+r) P(\eta))\right]
\end{aligned}
$$

The coefficient of $P^{\prime}(\eta)$ in L.H.S,

$$
\left\{u^{\prime \prime}\left(A^{p}-P(\eta)\right)+\left[(1-\eta) \beta(1+r)^{2} / \varphi\right] u^{\prime \prime}((1+r) P(\eta) / \varphi)+\varphi \eta \beta(1+r)^{2} u^{\prime \prime}((1+r) P(\eta))\right\},
$$

is clearly negative since $u^{\prime \prime}<0$. The sign of R.H.S is negative, zero and positive, corresponding to $\sigma>1, \sigma=1$ and $\sigma<1$, respectively. Replacing $P(\eta)$ with $P_{E}(\eta)$, we have completed the proof.

Proof of (iii): The proof is almost the same as proof of (i) except for maximizing (5.3) subject to (5.2) instead of (5.1). The first order condition for point $E^{\eta}$ is:

$$
\begin{aligned}
& -u^{\prime}\left(A^{p}-P(\eta)\right)+\beta(1+r)[(1-\varphi)+\varphi(1-\eta)] u^{\prime}((1+r) P(\eta)[(1-\varphi)+\varphi(1-\eta)] /(\varphi(1-\eta))) \\
& +\varphi \eta \beta(1+r) u^{\prime}((1+r) P(\eta))=0
\end{aligned}
$$

We get a following equation for comparative statics:

$$
\begin{aligned}
& P^{\prime}(\eta)\left\{u^{\prime \prime}\left(A^{p}-P(\eta)\right)\right. \\
& +\beta(1+r)^{2}\left([(1-\varphi)+\varphi(1-\eta)]^{2} /(\varphi(1-\eta))\right) u^{\prime \prime}((1+r) P(\eta)[(1-\varphi)+\varphi(1-\eta)] /(\varphi(1-\eta))) \\
& \left.+\varphi \eta \beta(1+r)^{2} u^{\prime \prime}((1+r) P(\eta))\right\} \\
& =\beta(1+r)^{2}[(1-\varphi)+\varphi(1-\eta)]\left((1-\varphi) /\left(\varphi(1-\eta)^{2}\right)\right) u^{\prime \prime}((1+r) P(\eta)[(1-\varphi)+\varphi(1-\eta)] /(\varphi(1-\eta))) \\
& -\varphi \beta(1+r) u^{\prime}((1+r) P(\eta))
\end{aligned}
$$

Considering $u^{\prime}>0, u^{\prime \prime}<0$, clearly, R.H.S is negative, and the coefficient of $P^{\prime}(\eta)$ in

\section{L.H.S,}

$$
\begin{aligned}
& \left\{u^{\prime \prime}\left(A^{p}-P(\eta)\right)\right. \\
& \left.+\beta(1+r)^{2}[(1-\varphi)+\varphi(1-\eta)]^{2} /(\varphi(1-\eta))\right] u^{\prime \prime}((1+r) P(\eta)[(1-\varphi)+\varphi(1-\eta)] /(\varphi(1-\eta))) \\
& \left.+\varphi \eta \beta(1+r)^{2} u^{\prime \prime}((1+r) P(\eta))\right\}
\end{aligned}
$$

is also negative. So, replacing $P(\eta)$ with $P_{E}(\eta)$, the proof is done. 
Proof of (ii): Since (5.3) must be constant subject to (5.1), we have:

$$
u\left(A^{p}-P(\eta)\right)+\varphi(1-\eta) \beta u((1+r) P(\eta) / \varphi)+\varphi \eta \beta u((1+r) P(\eta))=\text { Const. over } \eta
$$

The first order condition with regard to $\eta$ produces:

$$
\begin{aligned}
& P^{\prime}(\eta)\left\{-u^{\prime}\left(A^{p}-P(\eta)\right)+(1-\eta) \beta(1+r) u^{\prime}((1+r) P(\eta) / \varphi)\right. \\
& \left.+\varphi \eta \beta(1+r) u^{\prime}((1+r) P(\eta))\right\} \\
& =\varphi \beta[u((1+r) P(\eta) / \varphi)-u((1+r) P(\eta))]>0
\end{aligned}
$$

Evaluating (A3.5) at $\eta=0$,

$$
P^{\prime}(0)\left\{-u^{\prime}\left(A^{p}-P(0)\right)+\beta(1+r) u^{\prime}((1+r) P(0) / \varphi)\right\}>0
$$

So, we need the sign of the coefficient of $P^{\prime}(0)$ in L.H.S,

$$
\left\{-u^{\prime}\left(A^{p}-P(0)\right)+\beta(1+r) u^{\prime}((1+r) P(0) / \varphi)\right\} .
$$

But, this is exactly the first order condition at $E^{\eta=0}(=E)$, which should be 0 at $E$. Since $I^{\eta=0}(=I)$ is located in the left-down side of $E$ along $l_{1}$, so (A3.7) should have a positive value. The proof is now done.

Proof of (iv): Since (5.3) must be constant subject to (5.2), we have:

$$
\begin{aligned}
& u\left(A^{p}-P(\eta)\right)+\varphi(1-\eta) \beta u((1+r) P(\eta)[(1-\varphi)+\varphi(1-\eta)] /(\varphi \beta(1-\eta))) \\
& +\varphi \eta \beta u((1+r) P(\eta))=\text { Const. } \\
& \text { over } \eta
\end{aligned}
$$

Differentiating (A3.8) with regard to $\eta$ produces:

$$
\begin{aligned}
& P^{\prime}(\eta)\left\{-u^{\prime}\left(A^{p}-P(\eta)\right)\right. \\
& +\beta(1+r)[(1-\varphi)+\varphi(1-\eta)] u^{\prime}((1+r) P(\eta)[(1-\varphi)+\varphi(1-\eta)] /(\varphi(1-\eta))) \\
& \left.+\varphi \eta \beta(1+r) u^{\prime}((1+r) P(\eta))\right\} \\
& =\varphi \beta[u((1+r) P(\eta)[(1-\varphi)+\varphi(1-\eta)] /(\varphi(1-\eta)))-u((1+r) P(\eta))] \\
& +\beta(1+r)(1-\varphi) /(1-\eta) u^{\prime}((1+r) P(\eta)[(1-\varphi)+\varphi(1-\eta)] /(\varphi(1-\eta))) P(\eta)
\end{aligned}
$$

Evaluating (A3.9) at $\eta=0$,

$$
\begin{aligned}
& P^{\prime}(0)\left\{-u^{\prime}\left(A^{p}-P(0)\right)+\beta(1+r) u^{\prime}((1+r) P(0) / \varphi)\right\} \\
& =\varphi \beta[u((1+r) P(0) / \varphi)-u((1+r) P(0))] \\
& +\beta(1+r)(1-\varphi) u^{\prime}((1+r) P(0) / \varphi) P(0) \\
& >0
\end{aligned}
$$

Now we have only to examine the sign of the coefficient of $P^{\prime}(0)$ in L.H.S; 
$\left\{-u^{\prime}\left(A^{p}-P(0)\right)+\beta(1+r) u^{\prime}((1+r) P(0) / \varphi)\right\}$, which is exactly the same as (A3.7). Since $l_{1}^{\eta=0}=l_{1}$, we can apply the same argument as after (A3.7) in the proof of (ii). The proof is now done.

\section{Proof of Proposition 5:}

Proof of (i): Children's associated indirect utility including default risk is given in (5.6). Maximizing (5.6) subject to (5.4) with regard to $R$ (and implicitly $P$ ) and $c_{1}$, we have the first order condition, with regard to $R$, for some value of $c_{1}$ such that

$$
\begin{array}{r}
\hat{c}_{1}=\underset{c_{1}, P, R}{\arg \max } \widetilde{\widetilde{v}}^{c}\left(P, R, \varphi^{\prime}, \beta, \eta, r, \bar{W}^{c}\right) \text { s.t. }(5.4): \\
1-\eta=\frac{u^{\prime}\left((1+r)\left(\bar{W}_{1}^{c}-\frac{\left(1-\varphi^{\prime}\right)}{\varphi^{\prime}} R-\hat{c}_{1}\right)\right)}{u^{\prime}\left((1+r)\left(\bar{W}_{0}^{c}+R-\hat{c}_{1}\right)\right)}
\end{array}
$$

Since $u^{\prime}()$ is a decreasing function $\left(u^{\prime \prime}<0\right)$, for $\eta>0$ we have:

$$
\bar{W}_{1}^{c}-\frac{1-\varphi^{\prime}}{\varphi^{\prime}} R-\hat{c}_{1}>\bar{W}_{0}^{c}+R-\hat{c}_{1}
$$

So, we get $\varphi^{\prime}\left({\overline{W_{1}}}^{c}-\bar{W}_{0}^{c}\right)>R$ and $\left(1-\varphi^{\prime}\right)\left(\bar{W}_{1}^{c}-\bar{W}_{0}^{c}\right)>P$ (A3.13). Replacing $R$ and $P \quad$ with $\quad R^{c}(\eta)$ and $P^{c}(\eta)$, and considering $\quad R^{c}(0)=\varphi^{\prime}\left({\overline{W_{1}}}^{c}-\bar{W}_{0}^{c}\right)$ and $P^{c}(0)=\left(1-\varphi^{\prime}\right)\left({\overline{W_{1}}}^{c}-{\overline{W_{0}}}^{c}\right),\left(\right.$ A3.12) is equivalent with $R^{c^{\prime}}(0)<0, P^{c \prime}(0)<0$. The proof is now done.

Proof of (ii): Quite similar to proof of (i). Maximizing (5.6) subject to (5.5), in stead of subject to (5.4), with regard to $R$ (and implicitly $P$ ) and $c_{1}$, we have the first order condition, with regard to $R$, for some value of $c_{1}$ such that $\hat{c}_{1}=\underset{c_{1}, P, R}{\arg \max } \widetilde{\widetilde{v}}^{c}\left(P, R, \varphi^{\prime}, \beta, \eta, r, \bar{W}^{c}\right)$ s.t. (5.5): 


$$
1=\frac{u^{\prime}\left((1+r)\left(\bar{W}_{1}^{c}-\frac{\left(1-\varphi^{\prime}\right)(1-\eta)}{\varphi^{\prime}} R-\hat{c}_{1}\right)\right)}{u^{\prime}\left((1+r)\left(\bar{W}_{0}^{c}+R-\hat{c}_{1}\right)\right)}
$$

Then we have:

$\frac{\varphi^{\prime}}{\left(1-\varphi^{\prime}\right)(1-\eta)+\varphi^{\prime}}\left({\overline{W_{1}}}^{c}-{\overline{W_{0}}}^{c}\right)=R^{c}(\eta)$ and $\frac{\left(1-\varphi^{\prime}\right)(1-\eta)}{\left(1-\varphi^{\prime}\right)(1-\eta)+\varphi^{\prime}}\left({\overline{W_{1}}}^{c}-{\overline{W_{0}}}^{c}\right)=P^{c}(\eta)$

(A3.14). Considering $R^{c}(0)=\varphi^{\prime}\left({\overline{W_{1}}}^{c}-{\overline{W_{0}}}^{c}\right)$ and $P^{c}(0)=\left(1-\varphi^{\prime}\right)\left({\overline{W_{1}}}^{c}-{\overline{W_{0}}}^{c}\right), \quad$ (A3.14) implies $R^{c^{\prime}}(0)>0, P^{c}{ }^{\prime}(0)<0$. The proof is now done.

\section{Proof of Proposition 6:}

Proof of (i): Parents' associated indirect utility is given by (2.5a"). Maximizing (2.5a") subject to (5.8) with regard to $R$ (and implicitly $P$ ), we have the first order condition:

$$
\begin{aligned}
& -u^{\prime}\left(A^{p}-R \varphi /((1-\varphi)(1-\zeta))\right) \\
& +\beta(1+r)[\varphi+(1-\varphi)(1-\zeta)] u^{\prime}((1+r) R[\varphi+(1-\varphi)(1-\zeta)] /((1-\varphi)(1-\zeta)))=0
\end{aligned}
$$

Replacing $R$ with $R(\zeta)$ as a function of $\zeta$ in (A3.15), differentiating the equality with regard to $\zeta$, and implementing the comparative statics immediately produce the following equation:

$$
\begin{aligned}
& R^{\prime}(\zeta)\left\{(\varphi /((1-\varphi)(1-\zeta))) u^{\prime \prime}\left(A^{p}-R(\zeta) \varphi /((1-\varphi)(1-\zeta))\right)\right. \\
& \left.+\beta(1+r)^{2}\left([\varphi+(1-\varphi)(1-\zeta)]^{2} /((1-\varphi)(1-\zeta))\right) u^{\prime \prime}((1+r) R(\zeta)[\varphi+(1-\varphi)(1-\zeta)] /((1-\varphi)(1-\zeta)))\right\} \\
& =-\left(\varphi /\left((1-\varphi)(1-\zeta)^{2}\right)\right) R(\zeta) u^{\prime \prime}\left(A^{p}-R(\zeta) \varphi /((1-\varphi)(1-\zeta))\right) \\
& +\beta(1+r)(1-\varphi) u^{\prime}((1+r) R(\zeta)[\varphi+(1-\varphi)(1-\zeta)] /((1-\varphi)(1-\zeta))) \\
& -\beta(1+r)^{2}\left([\varphi+(1-\varphi)(1-\zeta)]^{2} \varphi /\left((1-\varphi)(1-\zeta)^{2}\right)\right) R(\zeta) u^{\prime \prime}((1+r) R(\zeta)[\varphi+(1-\varphi)(1-\zeta)] /((1-\varphi)(1-\zeta)))
\end{aligned}
$$

Considering $u^{\prime}>0, u^{\prime \prime}<0$, clearly, R.H.S is positive, and the coefficient of $R^{\prime}(\eta)$ in L.H.S is negative. So, replacing $R(\zeta)$ with $\widetilde{R}^{p}(\zeta)$, the proof is done.

Proof of (ii): Parents' associated indirect utility is given by (A2.5). Maximizing (2.5a") subject to (5.8) with regard to $P$ (and implicitly $R$ ), we have the first order condition: 


$$
\begin{aligned}
& -u^{\prime}\left(A^{p}-P\right) \\
& +\beta(1+r)[\varphi+(1-\varphi)(1-\zeta)] u^{\prime}((1+r) P[\varphi+(1-\varphi)(1-\zeta)] / \varphi)=0
\end{aligned}
$$

Replacing $P$ with $P(\zeta)$ as a function of $\zeta$ in (A3.17), differentiating the equality with regard to $\zeta$, and implementing the comparative statics immediately produce the following equation:

$$
\begin{aligned}
& P^{\prime}(\zeta)\left\{u^{\prime \prime}\left(A^{p}-P(\zeta)\right)\right. \\
& \left.+\beta(1+r)^{2}[\varphi+(1-\varphi)(1-\zeta)]^{2} u^{\prime \prime}((1+r) P(\zeta)[\varphi+(1-\varphi)(1-\zeta)] / \varphi)\right\} \\
& =\beta(1+r)(1-\varphi) u^{\prime}((1+r) P(\zeta)[\varphi+(1-\varphi)(1-\zeta)] / \varphi) \\
& +\beta(1+r)^{2}[\varphi+(1-\varphi)(1-\zeta)]((1-\varphi) / \varphi) P(\zeta) u^{\prime \prime}((1+r) P(\zeta)[\varphi+(1-\varphi)(1-\zeta)] / \varphi)
\end{aligned}
$$

Considering $u^{\prime \prime}<0$, clearly, the coefficient of $P^{\prime}(\eta)$ in L.H.S is negative. On the other hand, R.H.S can be rewritten as:

$$
\text { R.H.S }=\beta(1+r)(1-\varphi)\left(u^{\prime}(\mathrm{X})+\mathrm{X} u^{\prime \prime}(\mathrm{X})\right)
$$

where $\mathrm{X} \equiv(1+r)([\varphi+(1-\varphi)(1-\zeta)] / \varphi) P(\zeta)$

Considering the form of utility (2.1), the sign of R.H.S is positive, zero and negative,

corresponding to $\sigma<1, \sigma=1$ and $\sigma>1$, respectively. So, replacing $P(\zeta)$ with $\widetilde{P}^{p}(\zeta)$, the proof is done.

\section{Proof of Proposition 7:}

Proof of (i): Children's associated indirect utility including default risk is given in (2.5b'). Maximizing (2.5b') subject to (5.9) with regard to $R$ (and implicitly $P$ ) and $c_{1}$, we have the first order condition, with regard to $R$, for some value of $c_{1}$ such that

$$
\begin{aligned}
\hat{c}_{1}=\underset{c_{1}, P, R}{\arg \max } \tilde{v}^{c}\left(P, R, \varphi^{\prime}, \beta, r, \bar{W}^{c}\right) \text { s.t. (5.9): } \\
1-\zeta=\frac{u^{\prime}\left((1+r)\left(\bar{W}_{1}^{c}-\frac{\left(1-\varphi^{\prime}\right)}{\varphi^{\prime}(1-\zeta)} R-\hat{c}_{1}\right)\right)}{u^{\prime}\left((1+r)\left(\bar{W}_{0}^{c}+R-\hat{c}_{1}\right)\right)}
\end{aligned}
$$


Since $u^{\prime}()$ is a decreasing function $\left(u^{\prime \prime}<0\right)$, for $\eta>0$ we have:

$$
\bar{W}_{1}^{c}-\frac{\left(1-\varphi^{\prime}\right)}{\varphi^{\prime}(1-\zeta)} R-\hat{c}_{1}>\bar{W}_{0}^{c}+R-\hat{c}_{1}
$$

So, we get $\frac{\varphi^{\prime}(1-\zeta)}{\left(1-\varphi^{\prime}\right)+\varphi^{\prime}(1-\zeta)}\left({\overline{W_{1}}}^{c}-\bar{W}_{0}^{c}\right)>R$ (A3.22). L.H.S of (A3.22) is decreasing with regard to $\zeta$. Replacing $R$ with $\widetilde{R}^{c}(\zeta)$, and considering $\widetilde{R}^{c}(0)=\varphi^{\prime}\left({\overline{W_{1}}}^{c}-\bar{W}_{0}^{c}\right),(\mathrm{A} 3.22)$ is equivalent with $\widetilde{R}^{c}(0)<0$. The proof is now done. Unlike Proposition 5 , the sign of $\widetilde{P}^{c}(0)$ is still uncertain. 